\title{
Contextual data classification for a ubiquitous intelligent environment
}

\author{
Malika Yaici ${ }^{1}$. Salima Sabri ${ }^{2}$ Wissam Azni $^{3} \cdot$ Faiza Boudjemil $^{3}$
}

Received: 26 December 2019 / Accepted: 27 February 2020 / Published online: 4 March 2020

(c) Springer Nature Switzerland AG 2020

\begin{abstract}
Recognition of activities from sensors is a key paradigm of ubiquitous computing. Activity recognition systems can be used to label large sets of data. Variability in human activities, sensor deployment characteristics, and application domains has led to the development of best practices and methods to improve the robustness of activity recognition systems. Classification is one of the most important steps in making the recognition process more expressive and reducing uncertainty, thus minimizing representation. The K-medoid algorithm is simple but effective for grouping data according to the similarity that the samples present between them without the need to know each sample's membership class. In this paper, we propose a classification technique based on unsupervised partitioning algorithm, which allows recognizing activities and overcomes the problem of supervision.
\end{abstract}

Keywords Context data classification · Activity recognition $\cdot$ Clustering $\cdot \mathrm{K}$-medoids algorithm

\section{Introduction}

Thanks to the progress made in recent years in the miniaturization of electronic components and the emergence of wireless network technologies, a growing number of everyday objects incorporate electronic devices through which they become communicating. These objects are, depending on the case, either mobile or immobile. Finally, the user finds himself in the center of a space composed of heterogeneous physical objects, with or without wired communication capabilities. Such an environment that forms a spontaneous and dynamic federation of communicating objects is called "Ubiquitous" as defined, firstly, by Marc Weiser in 1991 [1].

One of the central elements of ubiquitous computing is the ability to recognize and understand its users dynamically. This ability can be produced by obtaining current and updated user information that represents its context. The user's context can be any information concerning his status, his location, his environment, his activity, etc. Context-aware computing is thus introduced to address the challenges of their recognition and understanding. Context recognition is the use of machine learning techniques to determine higher-level context descriptions from data collected from sensors. In our work, we are interested in a context related to the activity of a person.

Activity recognition is one of the issues of ubiquitous computing research and one of the most active research areas in ubiquitous systems. It is an essential element in the development of context-sensitive systems and data management systems [2].

The diversity in the nature of data and technologies deployed in ubiquitous environments greatly complicates the task of aggregating or interpreting data. The imperfection of the contextual information is generally due to their dynamicity which causes errors in the captured information, they can be thus, inconsistent, uncertain, incomplete or sometimes even contradictory. It is often necessary to set up pretreatment layers during activity recognition processes in order to transform these heterogeneous data into a representation mode allowing their joint exploitation [3].

$\triangle$ Malika Yaici, yaici_m@hotmail.com | 'Laboratoire LTII, University of Bejaia, Béjaïa, Algeria. ${ }^{2}$ Laboratoire LMI, University of Bejaia, Béjaïa, Algeria. ${ }^{3}$ Computer Department, University of Bejaia, Béjaïa, Algeria. 
Classification is one of the approaches to construct a model in the form of rules or decision criteria based on a priori knowledge of the class of samples provided by an expert. This model is then used to provide a decision about the membership of an unknown sample represented by its attributes to one of the predefined classes [4].

The aim of this research work consists in proposing a classification technique, non-supervised and easy to implement, based on a clustering algorithm: K-medoids.

This paper is organized around six sections. After this introduction, Sect. 2 provides an overview of Context and Context-awareness, and highlights activity recognition and classification. In Sect. 3 a state of the art of classification approaches for the recognition of existing human activities is given. Section 4 describes and explains our contribution and validated in Sect. 5. We finish this paper with a general conclusion and perspectives.

\section{Preliminaries notions}

\subsection{Context}

Context is an important concept for characterizing information about the user and his environment. Abowd et al. [5] retained as the most important elements to characterize the situation of an entity: time, localization, activity and identity. In addition, an application that uses the context must know when, and what (what the person is doing) to determine why a situation has occurred.

In this work, we use the same elements to compose the context, except the identity of the person who is not important since we assume the presence of only one person in the environment.

Context Aware Systems adapt their behavior according to several factors that condition their environment at a given moment, depending on the users, their location, accessible devices, but also according to the changes in the time these factors undergo. According to Dey et al. [6], a system is context aware if it uses the context to provide information or services relevant to the user. Context awareness is concerned with context acquisition (for example through sensors), abstraction and context understanding (by linking detected events with context elements), and adaptation of the system response based on the recognized context.

\subsection{Activity recognition}

Human Activity Recognition (HAR) from sensors is a scientific field in full swing that has emerged in research on human machine interactions and ubiquitous computing. Daily activities are contextual data about users and their use of essential equipment to help them and allow them to save energy.

The recognition of activities in a given space requires the interaction of computer systems with their environments. So, the installation of sensors, in a given environment, allows the latter to be monitored by providing accurate information. Processing this information gives a report of all the activities of people or moving objects in that environment.

\subsection{Classification}

Classification is the task of learning an objective function $f$ to assign a set of attributes $x$ to one of the predefined classes labelled $y$. The function $f$ can also be called a classification model. This classification model can be used in different situations [7]. The main purpose of the classification is to identify the classes to which objects belong from the descriptive traits, also called attributes, characteristics, or features. Usually, a probability is assigned to the output classes and can be used for decision merging [3].

The attributes in our case represent the events and the classes represent the activities.

Classification methods have one thing in common: the need to learn and evaluate their performance. They, therefore, require two sets of samples:

(a) a learning base: it must contain a sufficient number of samples $x_{i}$ of each class, this set must be as representative as possible from a qualitative point of view (to the intended application) and quantitatively (a large number of samples while respecting the prior probabilities of each class), and

(b) a test base: it is important to choose this set with rigor because it allows to evaluate the classification algorithm in question.

Typically, the process takes place in two main phases: learning (off-line) and decision (online). The learning module allows, when it is supervised, i.e. from the prior knowledge of the class of samples provided by an expert, to build a model (set of rules or decision criteria). This model is then used by the classifier to provide the decision regarding the membership of an unknown sample, represented by its attributes, to one of the predefined classes.

Depending on whether the class of learning samples is known or not, we obtain two major families of classification methods and therefore two main learning approaches [4]:

- Supervised Approaches: The general procedure for learning and testing a supervised learning algorithm 
for activity recognition usually takes place in the following five steps:

(a) acquiring activity sensor data including annotation of what the user is doing at the moment the acquisition of these data;

(b) transforming these data into characteristics, for example, by calculating specific properties, reducing dimensionality;

(c) divide this set of features into a learning set and a test set;

(d) learn the algorithm using the training set;

(e) test the performance of the algorithm classification using the test data set.

- Unsupervised approaches: In an unsupervised approach, learning consists in acquiring non-labelled data and transforming it into characteristics, then modelling it using a clustering algorithm. During partitioning each data point is assigned to one or $\mathrm{N}$ groups of points that are close to it according to a predefined distance measure.

\subsection{Classifier's performances evaluation}

Learning a classifier is like training a model on a learning set that minimizes the error rate which is the number of misclassified examples. The goal is to get a classifier that makes fewer mistakes if we introduce new cases that we did not watch during the training phase. There are several techniques for evaluating the performance of a classifier.

- Cross validation: In this type of validation, classification methods are evaluated on a test basis. There exist many techniques of cross-validation:

- Hold-out method: it consists of separating all the data available into two sets, a training set and a test set. Then train the model on the training set and evaluate it on the test set.

- K-fold-cross-validation: it consists of dividing the set of data of size $\mathrm{n}$ in $\mathrm{k}$ sub-bases $\mathrm{k}$ times. Then train the model on $k-1$ sub-bases, and then validate it on the $\mathrm{k}$ basis. The procedure is repeated $\mathrm{k}$ times.

- Confusion matrix: A binary classifier produces output with two class values or labels, such as Yes/No, for given input data. The class of interest is usually denoted as 'positive' and the other as 'negative'. Let TP, FP, TN, FN respectively represent the number of correctly recognized positives, the number of negatives detected by error, the number of negatives correctly recognized, the number of positives detected by mistake, then the Con- fusion matrix of binary classification will be as shown on Table 1.

\section{Related works}

In this section we will present the main works found in the field of activity recognition literature.

In [8], a comparison of several classification and automatic feature selection algorithms is undergone. The contribution of this study is to find out the advantages in using sophisticated and complex classification methods compared with a simple method that can easily be implemented in mobile devices. The main result is that "even a simple linear classification algorithm can achieve a reasonably good accuracy if the features calculated from raw data are selected in a suitable way" [8].

In general, the application of Bayes statistical analysis to classification problems is based on probabilistic class modelling. In [9], a naïve Bayes classifier is used to early gesture recognition. The naïve baseline is based on three main observations: (1) the effectiveness of the naïve Bayes classifier in text mining problems; (2) the link between natural language processing and computer vision via the bag-of-words representation; and (3) the cumulative-evidence nature of the inference process of naïve Bayes.

The $\mathrm{K}$ nearest neighbor algorithm is based on the concept of proximity. The nearest neighbor $\mathrm{K}$ algorithm defines a function of distance between feature vectors to classify a new entry. The choice of this distance function is essential to the proper functioning of the method. In [10], privacy issues due when using external activity recognition services are addressed. A privacy-preserving version of the K Nearest Neighbors (K-NN) classifier is proposed and is based on the (II-CSP+) cryptography-free private similarity evaluation protocol.

The support vector machine (SVM) is an algorithm that uses a non-linear function to transform the original data into a high dimension. In [11], multi-class activity classification problem is addressed and a hierarchical approach based on a robust least squares twin SVM algorithm is proposed. This algorithm handles the heteroscedastic noise and outliers present in activity recognition framework.

Hidden Markov Model (HMM) is characterized by a dual stochastic process: an internal process that models the unobservable states $X=\left\{x_{1}, x_{2}, \ldots, x_{N}\right\}$ of a system (which is supposed to be a Markovian process) and an external

Table 1 Confusion matrix

\begin{tabular}{lll}
\hline & Predicted positive & Negative \\
\hline Observed positive & TP & FP \\
Negative & FN & TN \\
\hline
\end{tabular}


process that models the observations $Y=\left\{y_{1}, y_{2}, \ldots, y_{N}\right\}$ of the system whose states are hidden. In [12], the development of a Human Activity Recognition and Segmentation (HARS) system based on HMMs, with the incorporation of an activity sequence model, is described. This system uses inertial signals from a smartphone to recognize and segment six different physical activities: walking, walking-upstairs, walking-downstairs, sitting, standing and lying down.

Many neural networks are currently known and exploited. In [13], and according to the authors, they are the first to apply recurrent neural networks to the activity recognition task. Recurrent neural networks are applied to the task of recognizing the surgical activities of the kinematics of the robot. They are working on the recognition of gestures and higher-level activities or maneuvers, and modelling the kinematics map of gestures / maneuvers with recurrent neural networks using a single model and a single set of hyper-parameters.

Khaire et al. [14] proposed a ConvNets based approach for activity recognition by combining multiple deep learning methods's cues. A new method of creating skeleton images, from skeleton joint sequences, representing motion information is also presented in this paper. Motion representation images, namely, Motion History Image (MHI), Depth Motion Maps (DMMs) and skeleton images are constructed from RGB, depth and skeletal data of RGB-D sensor. A separate training of these images on ConvNets is then undergone and respective softmax scores are fused at the decision level.

Decision trees are one of the most well known and used techniques in classification due in part to their ability to deal with complex classification problems. Activity recognition systems may produce false classes when trained models deal with real-world behavior. Two reasons can lead to misclassifications. First, the trained model may be overfit, incorporating noise from the training data. Second, the model may be insufficient, producing spurious classifications for real-world behavior. In [15], the authors pruned a trained $\mathrm{C} 4.5$ decision tree model by removing the redundant leaf labels that tend to produce misclassifications using a thresholding technique.

Several context-aware systems use ontologies to represent context. iKnow, an ontology-driven framework for semantic situation understanding in pervasive multisensor environments for human activity recognition is presented in [16]. It uses OWL ontological knowledge to capture domain relationships between low-level observations and high-level activities, while context aggregation and activity interpretation are supported through contextaware fusion.

The state-of-the-art analysis of classification techniques for activity recognition has revealed that the most used techniques are supervised. This supervision requires the use of expensive and labelled collections of large learning sets. Then, they have the disadvantage of modelling according to the class of membership of each sample, which requires referring to the training set during the construction process of the classification model. Previous work has shown that it is possible to recognize human activities based on unsupervised or semi-supervised models. Our proposal stems from this idea in order to solve the problem of modelling according to the class of membership, we use the algorithm K-Medoids as simple but effective algorithm of data grouping in order to partition all the samples in groups according to the similarity that these samples have between them and without the need to know the class of membership of each sample. The result of this grouping is a set of partitions, each one of them is represented by the most central sample called representative sample, then, it is enough to attribute a label to one partition.

K-medoids and K-means clustering algorithms have already been used for big-data analysis (data-dmining) [17-19]. Park and Jun [17] proposed a new algorithm for K-medoids clustering, which behaves like the K-means algorithm and uses several methods for selecting initial medoids. The proposed algorithm calculates the distance matrix once and uses it for finding new medoids at every iterative step. The authors showed that, basing on the experimental results, the proposed algorithm reduces computation time, significantly, if compared to the partitioning around medoids.

The authors in [20] compared the two clustering algorithms K-means and K-medoids, and the results showed that K-medoids is better than K-means regarding the cluster head selection time and cluster overlapping space complexity. Also, they showed that $\mathrm{K}$-medoids is better in terms of execution time, non sensitive to outliers, reduces noise and minimizes the sum of dissimilarities of data objects. Lampert et al. [21] also showed the limits of the $\mathrm{K}$-means algorithm under some constraints.

To our knowledge, K-medoids (or K-means) algorithm has never been used in context data classification for activity recognition.

\section{Proposition}

The proposed classification technique is to be used in two main stages: the learning and the classification, of which a small preview is given below before entering the details of each phase:

- Learning: it is the step of building a model that will be exploited for classification. It consists in clustering the set 
of activity vectors $x$ using the K-medoid algorithm, then in cluster annotating;

- Classification: at this level, the exploitation of the model built during the learning step is done; the classification is carried out by the algorithm $\mathrm{K}$ nearest neighbors (K-NN).

\subsection{Dataset}

The data set used to train and validate the proposed classification technique for activity recognition is a data set generated in 2008 during an experiment in the DOMUS laboratory [22] where people had to wake up, go to the toilet, prepare breakfast, eat and wash the dishes. In this work, we will only consider the two activities (wake up) and (eat) for simplification reasons.

The experiment was carried out with six different people and generated 444 activity instances, one instance corresponds to a realization, by a person, of a particular activity. The number of instances, corresponding to the two activities we have chosen, is 160 instances. These 160 instances collected were divided into two series. The first series (contains 110 instances) corresponds to the first time that people performed these two activities, it was used for learning, because 110 instances spread over two different activities is more than enough to make learning. The second series (contains 50 activity instances) is the second time that users have done both activities and has been used for validation.

The dataset used to train the classification technique consists of two files: the first is generated automatically by the server and contains all the events of the sensors generated with the precise time of their triggering. The second is an annotations file, filled "by hand" by a person who has analyzed the activities carried out and has indicated for each of them its start and end time. For example, according to the annotation file, activity 1 , which corresponds to the "wake up" activity, was performed between 09:17:46 and 09:18:00 (Table 2). Referring to the second file, the sensor events file (Table 3), it is possible to know which events were generated during the execution of this activity, i.e. the sensors 5105, 0215 and 5101 [22].

\subsection{Learning}

The stages of learning an activity $A$ are:

Table 2 Example of an annotation file

\begin{tabular}{lll}
\hline Start time & End time & ID \\
\hline 11-Aug-2008 09:17:46 & 11-Aug-2008 09:18:00 & 1 \\
11-Aug-2008 09:18:04 & 11-Aug-2008 09:18:51 & 2 \\
\hline
\end{tabular}

Table 3 Example of a sensor event file

\begin{tabular}{llllll}
\hline Date & Time & ID & Type & Room & State \\
\hline 2008-08-11 & $9: 17: 46$ & 5105 & Light & Bedroom & Open \\
2008-08-11 & $9: 17: 48$ & 0215 & Door & Bedroom & Open \\
2008-08-11 & $9: 17: 53$ & 5101 & Light & Kitchen & Open \\
2008-08-11 & $9: 17: 57$ & 5105 & Light & Bedroom & Close \\
2008-08-11 & $9: 18: 0$ & 0215 & Door & Bedroom & Close \\
2008-08-11 & $9: 18: 4$ & 0216 & Door & Bathroom & Open \\
2008-08-11 & $9: 18: 5$ & 5106 & Light & Bathroom & Open \\
2008-08-11 & $9: 18: 9$ & 0216 & Door & Bathroom & Close \\
2008-08-11 & $9: 18: 12$ & F101 & Water & Bathroom & Open \\
\hline
\end{tabular}

1. Construction of the activity vectors $x$ from the dataset. They contain binary values indicating whether a sensor is activated or not during the execution of the given activity.

2. Partitioning of all these activity vectors into $k$ partitions using the K-Medoids algorithm.

3. The annotation: consists in attributing to the cluster (representative vector) a label.

Figure 1 shows the general diagram of the learning operation.

\subsubsection{Construction of activity vectors}

The input data of our algorithm, obtained from the previously discussed data set, are vectors indicating whether or not a sensor is enabled. Each time an activity is performed, we obtain an instance of this activity, which gives a vector of activity. We represent a vector of activity by $x$ whose dimension $d$ corresponds to the number of sensors. A large number of sensors therefore lead to a large dimension. To avoid this, it is necessary to select the most significant and important sensors, that is to say the best located and most differentiable. In our case, we will be interested in only five of them, these selected sensors correspond to the sensors that are activated during the realization of the activities that we considered in the context of this work: wake up and eat.

$x=\left(\begin{array}{l}0 \\ 1 \\ 0 \\ 1 \\ 1\end{array}\right)$ which means $\left(\begin{array}{lc}\text { sensor sens1: } & \text { non-activated } \\ \text { sensor sens2: } & \text { activated } \\ \text { sensor sens3: } & \text { non-activated } \\ \text { sensor sens4: } & \text { activated } \\ \text { sensor sens5: } & \text { activated }\end{array}\right)$

where: 
Fig. 1 Overall learning operation

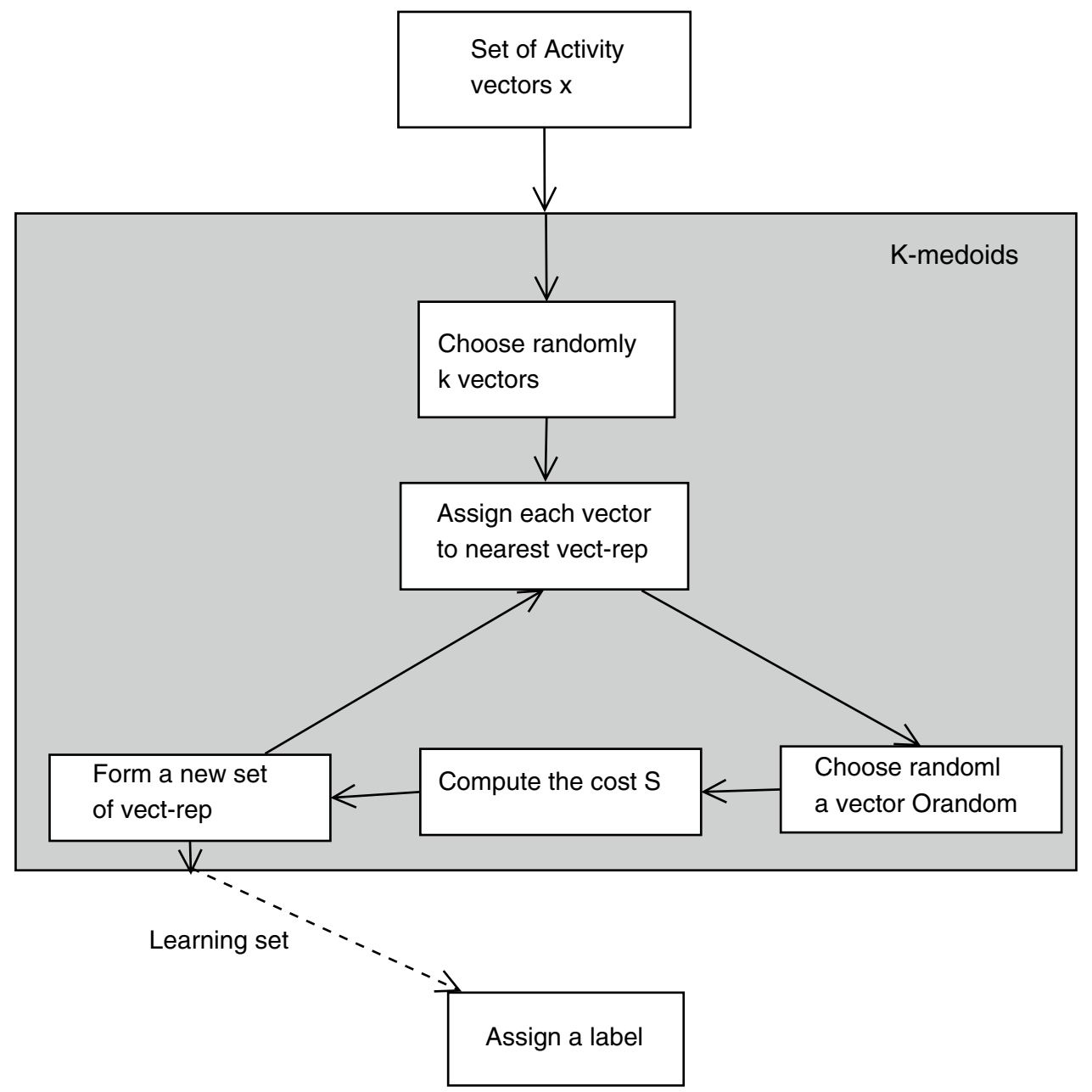

- sens1: is a pressure sensor placed on the door of the bedroom, to get information on the opening and closing of the door;

- sens2: is a light switch sensor placed on the light switch of the bedroom, it indicates whether the light is on or off;

- sens3: is an infrared sensor placed in the kitchen, it can detect the presence of a person in the kitchen;

- sens4: is a contactor for closet placed in the closet of the kitchen, it is activated when opening or closing this closet;

- sens5: is a flow meter placed on the faucet of the kitchen sink that provides information on the opening or closing of the faucet.

Remark 1 At this stage of our research only single activities performed by a unique or multiple actors are studied.

\subsubsection{Activity vectors partitioning}

K-means clustering is known to be sensitive to the outliers although it is quite efficient in terms of the computational time. For this reason, K-medoids clustering is chosen, because it is based on the most centrally located object in a cluster, it is less sensitive to outliers in comparison with the $\mathrm{K}$-means clustering. The objective is to use K-medoids (Algorithm 1) in order to partition the set of activity vectors $x$, so the set of these vectors will be provided as input to this algorithm, and it will return the corresponding clusters and their representative vectors. Among many algorithms for K-medoids clustering, partitioning around medoids (PAM) is known to be most powerful [17].

Instead of taking the average value of the examples in a cluster as a reference point as in the case of the K-means algorithm, we can take the examples to represent the clusters using a representative example by cluster, each of the 
remaining examples is clustered with the representative example for which it is most similar. The clustering method is thus performed based on the principle of minimizing the sum of the di-similarities between each object and its corresponding reference point. The absolute error criterion is used and which is defined as follows:

$E=\sum_{j=1}^{k} \sum_{p \in C_{j}}\left|p-o_{j}\right|$

where $E$ is the sum of the absolute errors of all the examples in the dataset, $p$ is a point in space that represents a given example in a cluster $C_{j}$, and $o_{j}$ is the representative example of $C_{j}$.

Let's take a closer look at K-Medoid clustering. The initial representative examples are chosen arbitrarily, the iterative process of replacing the representative examples with non-representative examples continues as long as the resulting clustering is improved. This quality is estimated by using the cost function which measures the average di-similarity between an example and the representative example of its cluster, the cost function is obtained by making the difference between the absolute error at iteration $i$ and the absolute error at iteration $i+1$.

To determine if a non-representative example $O_{\text {random }}$ is a good replacement for representative example, $O_{j}$, the following four cases are examined for each of the nonrepresentative examples $p$.

- Case1: $p$ currently belongs to the representative example $O_{j}$. If $O_{j}$ is replaced by $O_{\text {random }}$ as a representative example and $p$ is the closest to one of the other representative examples, $O_{i}, i \neq j$, then $p$ is reassigned to $O_{i}$.

- Case2: $p$ currently belongs to the representative example $O_{j}$. If $O_{j}$ is replaced by $O_{\text {random }}$ as a representative example and $p$ is the closest to $O_{\text {random }}$ then $p$ is reassigned to $O_{\text {random }}$.

- Case3: $p$ currently belongs to the representative example $O_{i}, i \neq j$. If $O_{j}$ is replaced by $O_{\text {random }}$ as a representative example and $p$ is always closer to $O_{i}$ then the assignment does not change.

- Case4: $p$ currently belongs to the representative example $O_{i}, i \neq j$. If $O_{j}$ is replaced by $O_{\text {random }}$ as a representative example and $p$ is closer to $O_{\text {random }}$ then $p$ is reassigned in $O_{\text {random }}$.

Whenever a reassignment takes place, a difference in the absolute error is added to the cost function. Therefore, the cost function calculates the difference in the value of the absolute error if the current representative example is replaced by a non-representative example. The total cost of permutations is the sum of the costs incurred by all non-representative examples. If the cost is negative then the example is replaced or permuted with $O_{\text {random }}$ so that the absolute error $E$ is reduced. If the total cost is positive then the current representative example $O_{j}$ is considered acceptable, and no changes in this iteration will be made.

PAM (Partitioning Around Medoids) [17] was one of the first introduced K-Medoid algorithms, trying to determine $K$ partitions for $n$ examples. After an initial random selection of representative $K$ examples, the algorithm repetitively attempts to make the choice of representative examples better. All possible pairs of examples are analyzed, such that an example in each pair is considered the representative example and the other is not. An example $O_{j}$ is replaced with the example which causes a big reduction in the error. The set of best examples for each cluster in an iteration forms the representative examples of the next iteration. The final set of representative examples are the respective medoids of the clusters.

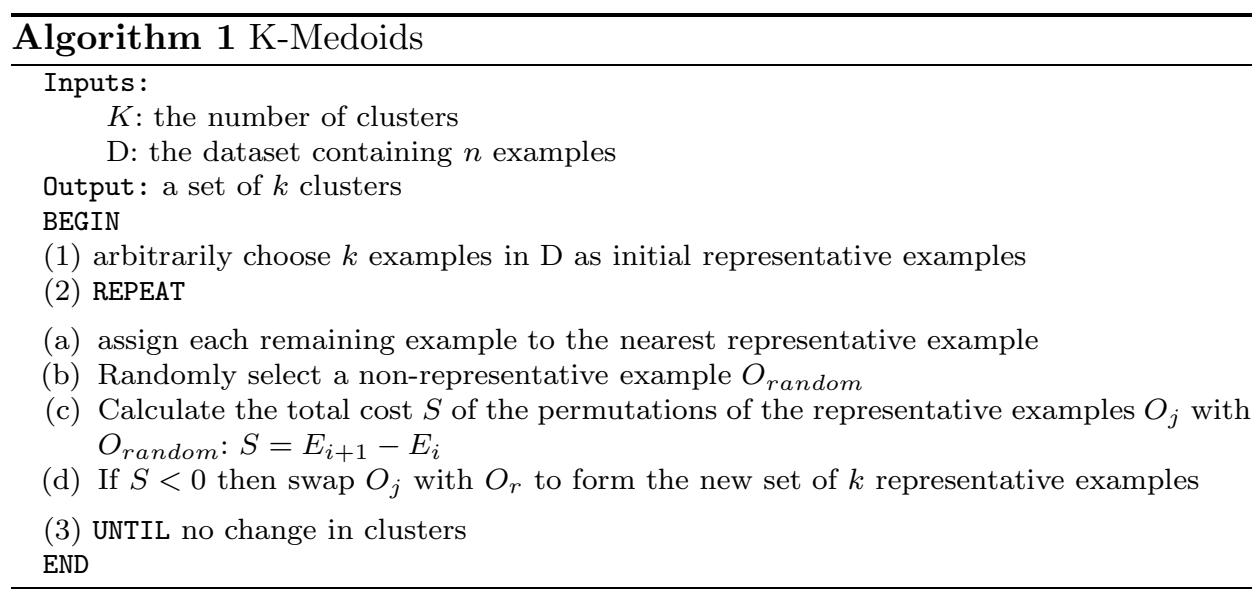


Table 4 Example of a learning base

\begin{tabular}{llllll}
\hline Sens1 & Sens2 & Sens3 & Sens4 & Sens5 & Label \\
\hline 1 & 1 & 0 & 0 & 0 & Wake up \\
0 & 0 & 1 & 1 & 0 & Eat breakfast \\
1 & 1 & 0 & 1 & 0 & Eat breakfast \\
1 & 1 & 0 & 0 & 1 & Wake up \\
0 & 1 & 1 & 0 & 1 & Wake up \\
1 & 1 & 0 & 1 & 1 & Eat breakfast \\
0 & 1 & 1 & 1 & 1 & Eat breakfast \\
1 & 1 & 1 & 0 & 0 & Wake up \\
0 & 0 & 1 & 1 & 1 & Eat breakfast \\
1 & 1 & 1 & 0 & 0 & Wake up \\
\hline
\end{tabular}

\subsubsection{Cluster annotation}

Annotation is the task of assigning each cluster a label. The label is actually assigned to the representative sample of the cluster, since this example is the most central one, or otherwise expressed, it represents the other examples found within this cluster. To assign a label to a cluster, we refer to the learning set that contains the activity vectors we have partitioned and their associated labels. Table 4 shows an example of a learning base.

A parsing of the learning base is performed for each representative vector. If the vector is found then the cluster it represents is labelled by the corresponding label, otherwise the label of the most similar vector within the cluster which appears also in the learning base will be adopted.

The annotation procedure is given in algorithm 2 .

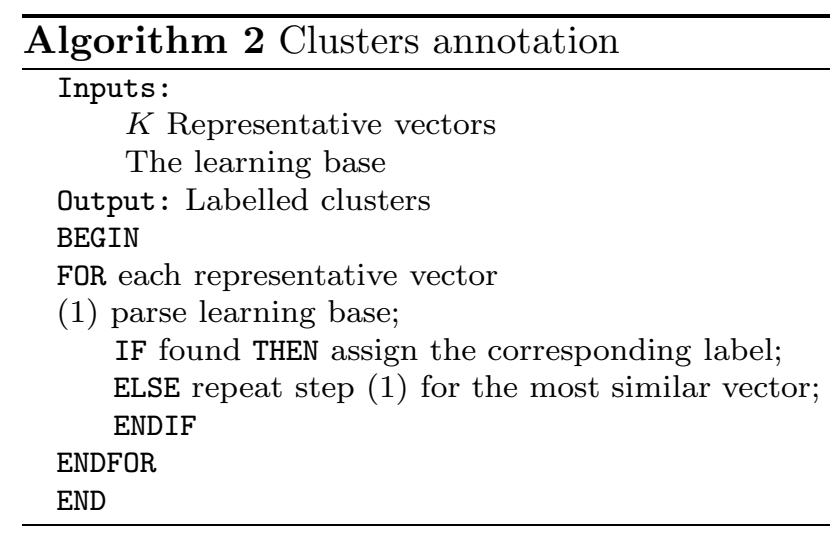

Remark 2 For binary vectors at most two parsing of the learning base will be executed.This method of cluster annotation has not been evaluated as it is not the objective of the paper.

\subsection{Classification}

The classification consists of using the model learned during the learning process to classify a new element (activity vector) whose label is unknown. The classification, based on K-NN algorithm, is performed by assigning to the test vector the label of the nearest representative vector. Euclidean distance is used and defined as follows:

$D(X, Y)=\sqrt{\sum_{i=1}^{n}\left(X_{i}-Y_{i}\right)^{2}}$

$X=\left(x_{1}, x_{2}, \ldots x_{n}\right)$ is the test vector whose label is to be found; $Y=\left(y_{1}, y_{2}, \ldots y_{n}\right)$ is the representative vector. The classification process, therefore, is as shown in Algorithm 3.

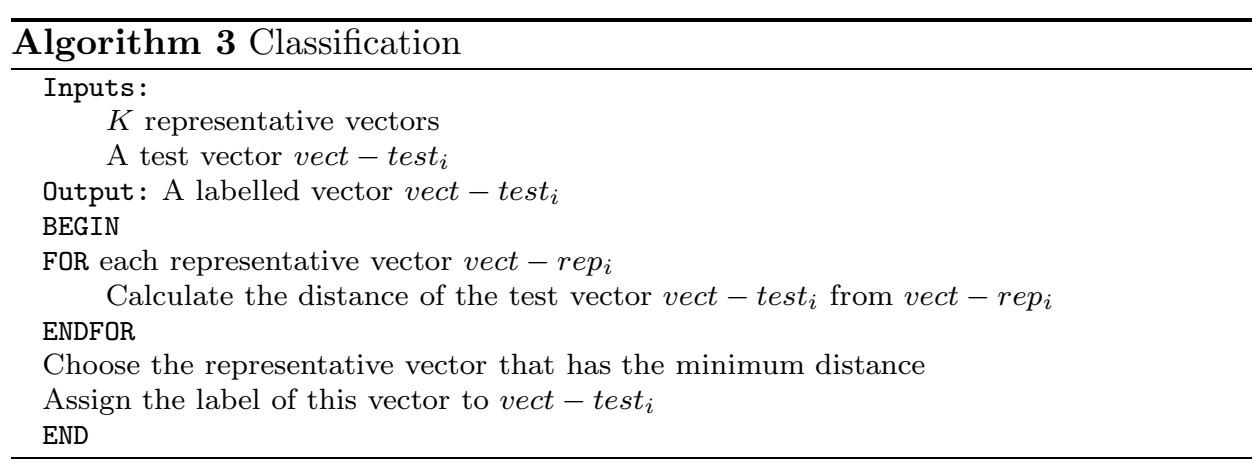


The algorithm will have as inputs the representative vectors of the clusters, obtained by applying the K-Medoid algorithm, and the test vector for which the label is sought, these representative vectors are the $k$ nearest neighbors. It, then, proceeds to calculate the distances between each representative vector and the test vector. Then, it assigns the test vector the label of the representative vector that has the minimum distance.

\section{Validation}

To validate our proposition, the hold-out cross validation method is used. The proposed classification technique is applied to the data set presented in Sect. 4.1, and more specifically to the set of test vectors that contains 50 instances of labelled activities, the obtained results are presented in Table 5.

The confusion matrix associated with our classification technique is given in Table 6 .

With the confusion matrix, the following performance measures are computed:

- Accuracy: $A C C=\frac{T P+T N}{T P+F P+F N+T N}=\frac{42}{49}=0.8571$

- Sensitivity (Recall): $S N=\frac{T P}{I P+F N}=\frac{20}{20}=1$

- Specificity: $S P=\frac{T N}{T N_{T} F P}=\frac{22}{29}=0.7586$

- Precision: $P R E=\frac{T N_{T P} F P}{T P+F P}=\frac{20}{27}=0.7407$

The results are interesting, since $85.71 \%$ (ACC) of the activities are recognized correctly. The best measures for PRE, SN or SP is 1.0, and in our case the measures may be qualified as good.

The results obtained after applying the proposed method to the whole set of data (series 1 and series2: 444 instances) in order to recognize the five activities that have been annotated during the experiment are given in Table 7.

Table 5 Validation results of the proposed classification technique

\begin{tabular}{lll}
\hline Activities & Total number of instances & $\begin{array}{l}\text { Number of } \\
\text { recognized } \\
\text { instances }\end{array}$ \\
\hline Wake up & 28 & 20 \\
Eat & 22 & 22 \\
\hline
\end{tabular}

Table 6 Confusion matrix for the two activities wake-up and eat
Table 7 Validation results of the proposed classification technique

\begin{tabular}{llll}
\hline Activities & $\begin{array}{l}\text { Total number } \\
\text { of instances }\end{array}$ & $\begin{array}{l}\text { Number of } \\
\text { recognized } \\
\text { instances }\end{array}$ & $\begin{array}{l}\text { Recognition } \\
\text { accuracy (\%) }\end{array}$ \\
\hline Wake up & 76 & 70 & 92.10 \\
Use toilet & 94 & 88 & 93.62 \\
Prepare breakfast & 91 & 85 & 93.40 \\
Have breakfast & 84 & 80 & 95.24 \\
Wash dishes & 99 & 92 & 92.93 \\
Average accuracy & & & 93.46 \\
\hline
\end{tabular}

Table 8 Validation results obtained by Chikhaoui et al. [23]

\begin{tabular}{ll}
\hline Experiments & $\begin{array}{l}\text { Average recog- } \\
\text { nition accuracy } \\
(\%)\end{array}$ \\
\hline Series1 episode length $N=3$ & 86.08 \\
Series 1 episode length $N=2$ & 92.28 \\
Series2 episode length $N=3$ & 90.41 \\
Series2 episode length $N=2$ & 95.26 \\
\hline
\end{tabular}

These results can be compared to the results given in Table 8 obtained by Chikhaoui et al. [23] where the same experimental data set is used to validate their proposal based on frequent pattern mining (semi-supervised). The annotated sensor data is used to built patterns for each model of activity then a mapping function is used to compare frequent patterns and models.

The two activities which were hard to recognize are preparing breakfast and washing dishes because similar sensors are involved and activity overlapping was possible.This can be avoided by a more sophisticated vector activity format. Taking into account, in addition to sensors states, time and activity duration can help.

\section{Conclusion}

We have shown through the state of the art that on the one hand the existing classification methods are supervised, and on the other hand, existing models are difficult to implement. This has allowed us to reduce supervision, highlighting the need to address these issues in a general way.

The idea of our contribution was not only to show that it was important to reduce supervision, but also to provide a classification method that is easy to implement and realize. The results obtained in the validation phase are promising. This work opens up for some perspectives to improve our classification technique such as multiple 
activities by multiple users and completely getting rid of supervision.

\section{Compliance with ethical standards}

Conflict of interest On behalf of all authors, the corresponding author states that there is no conflict of interest.

\section{References}

1. Weiser M (1991) The computer for the 21st century. Sci Am 265(3):94-104

2. Twinanda A-P (2017) Vision-based approaches for surgical activity recognition using laparoscopic and RGBD videos. University of Strasbourg, Strasbourg PhD thesis

3. Roggen D, Magnenat S, Waibel M, Tröster G (2011) Wearable computing : designing and sharing activity-recognition systems across platforms. IEEE Robot Autom Mag 18:83-95. https ://doi.org/10.1109/MRA.2011.940992

4. Sabri S (2011) Application de la Théorie des Jeux pour la Technique de Clustering en Data Mining. University of Bejaia, Bejaïa PhD thesis

5. Abowd GD, Dey AK, Brown PJ, Davies N, Smith M, Steggles P (1999) Towards a better understanding of context and context-awareness. In: Gellersen HW (ed) Handheld and ubiquitous computing, 2nd edn. Springer, Berlin, pp 304-307

6. Dey AK (2001) Understanding and using context. Pers Ubiquitous Comput 5(1):47

7. Charfi I (2013) Détection automatique de chutes de personnes basée sur des descripteurs spatiotemporels : définition de la méthode, évaluation des performances et implantation temps réel. PhD thesis, University of Bourgogne, France

8. Könönen V, Mäntyjärvi J, Similä H, Pärkkä J, Ermes M (2010) Automatic feature selection for context recognition in mobile devices. Pervasive Mob Comput 6:181-197. https://doi. org/10.1016/j.pmcj.2009.07.001

9. Escalante HJ, Morales EF, Sucar LE (2016) A naive Bayes baseline for early gesture recognition. Pattern Recognit Lett 73:9199. https://doi.org/10.1016/j.patrec.2016.01.013

10. Gheid Z, Challal Y, Yi X, Derhab A (2017) Efficient and privacyaware multi-party classification protocol for human activity recognition. J Netw Comput Appl 98:84-96. https://doi. org/10.1016/j.jnca.2017.09.005

11. Khemchandani R, Sharma S (2016) Robust least squares twin support vector machine for human activity recognition. Appl Soft Comput 47:33-46. https://doi.org/10.1016/j. asoc.2016.05.025

12. San-Segundo R, Lorenzo-Trueba J, Martinez-Gonzalez B, Pardo JM (2016) Segmenting human activities based on HMMs using smartphone inertial sensors. Pervas Mob Comput J 30:84-96. https://doi.org/10.1016/j.pmcj.2016.01.004

13. DiPietro R, Lea C, Malpani A, Ahmidi N, Vedula SS, Lee Gl, Lee MR, Hager GD (2016) Recognizing surgical activities with recurrent neural networks. In: Proceedings of international conference on medical image computing and computer-assisted intervention (MICCAI), Athens, Greece, pp 551-558. https://doi. org/10.1007/978-3-319-46720-7_64

14. Khaire $P$, Kumar $P$, Imran J (2018) Combining CNN streams of RGB-D and skeletal data for human activity recognition. Pattern Recognit Lett 115:107-116. https://doi.org/10.1016/j.patre c.2018.04.035

15. Phan T (2014) Improving activity recognition via automatic decision tree pruning. In: Proceedings of international joint conference on pervasive and ubiquitous computing: ADJUNCT, Seattle, WA, USA, pp 827-831. https://doi.org/10.1145/26387 28.2641310

16. Núñez JC, Cabido R, Pantrigo JJ, Montemayor AS, Vélez JF (2018) Convolutional neural networks and long short-term memory for skeleton-based human activity and hand gesture recognition. Pattern Recognit 76:80-94. https://doi.org/10.1016/j.patco g.2017.10.033

17. Park H-S, Jun C-H (2009) A simple and fast algorithm for K-medoids clustering. Expert Syst Appl 36(2):3336-3341. https ://doi.org/10.1016/j.eswa.2008.01.039

18. Soheily-Khah S, Douzal-Chouakria A, Gaussier E (2016) Generalized $\mathrm{k}$-means-base d clustering for temporal data under weighted and kernel time warp. Pattern Recognit Lett 75:63-69. https://doi.org/10.1016/j.patrec.2016.03.007

19. Mavroeidis D, Marchiori E (2014) Feature selection for k-means clustering stability: theoretical analysis and an algorithm. Data Min Knowl Discov 28(4):918-960. https://doi.org/10.1007/s1061 8-013-0320-3

20. Arora P, Deepali D, Varshney S (2016) Analysis of K-means and K-medoids algorithm for big data. Procedia Comput Sci 78:507512. https://doi.org/10.1016/j.procs.2016.02.095

21. Lampert T, Dao TBH, Lafabregue B, Serrette N, Forestier G, Crémilleux B, Vrain C, Gançarski P (2018) Constrained distance based clustering for time-series: a comparative and experimental study. Data Min Knowl Discov 32(6):1663-1707. https://doi. org/10.1007/s10618-018-0573-y

22. Domus Data https://www.usherbrooke.ca/domus/fr/recherche/ jeux-de-donnees/; http://domuslab.fr/datasets/. Accesse 11 Feb 2020

23. Chikhaoui B, Wang S, Pigot $H$ (2011) A frequent pattern mining approach for ADLs recognition in smart environments. In: Proceedings of IEEE international conference on in advanced information networking and applications (AINA), Singapore, pp 248-255. https://doi.org/10.1109/AINA.2011.13

Publisher's Note Springer Nature remains neutral with regard to jurisdictional claims in published maps and institutional affiliations. 